\title{
Cold H I in IRC +10216
}

\author{
T. Le Bertre ${ }^{1}$ and E. Gérard ${ }^{2}$ \\ 1 DEMIRM, UMR 8540, Observatoire de Paris, 61 av. de l'Observatoire, 75014 Paris, France \\ 2 ARPEGES, UMR 8644, Observatoire de Paris, 5 place J. Janssen, 92195 Meudon Cedex, France \\ e-mail: Eric.Gerard@obspm.fr \\ Received 13 August 2001 / Accepted 6 September 2001

\begin{abstract}
With the recently refurbished Nançay Radiotelescope we have observed the H I $21 \mathrm{~cm}$ line in the direction of IRC +10216 , a typical high mass-loss carbon star. We found HI in absorption indicating that the expanding circumstellar gas may reach very low temperatures $(\leq 4 \mathrm{~K})$. The atomic gas could be the product of the photodissociation of molecular hydrogen in the outer envelope or result from a previous phase $\left(\geq 6 \times 10^{3}\right.$ years ago $)$ of mass loss when the star had a higher effective temperature than now.
\end{abstract}

Key words. stars: circumstellar matter - stars: mass-loss - stars: AGB and post-AGB - radio lines: stars

\section{Introduction}

Radio and infrared observations show that stars on the Asymptotic Giant Branch (AGB) loose matter at a large rate. The ejection of their external layers affects the future evolution of these objects, and for instance prevents that those with an initial mass larger than $1.4 M_{\odot}$ evolve to supernovae. Through the recycling of matter in the Interstellar Medium, this phenomenon is also important for the evolution of stellar systems (Olofsson 1999).

However the observations are difficult to interpret. The mass loss rate is variable with timescales ranging from a few years to several thousands years. Also most of the observations are sensitive to minor species, e.g. CO or dust, which represent less than $2 \%$ of the material flowing outwards. The mass loss estimates rely on the modelling of the emission from these species and on a conversion ratio which is uncertain. Most of the material is made of hydrogen, but this element has been detected in only a few sources.

We started a search for H I in circumstellar envelopes with the Nançay Radiotelescope which has been recently refurbished. We report here on the first detection of $\mathrm{HI}$ in the prototypical mass-losing AGB source, IRC +10216 .

\section{2. $\mathrm{HI}$ in circumstellar envelopes}

Most of the mass in the winds from red giants should be in hydrogen, either in atomic $(\mathrm{HI})$, ionised $\left(\mathrm{H}^{+}\right)$or molecular form $\left(\mathrm{H}_{2}\right)$. Glassgold \& Huggins (1983) have studied the distribution of atomic and molecular hydrogen in the circumstellar envelopes of cool giants. In the stellar atmospheres of stars with effective temperature, $T_{\star}$, lower than

Send offprint requests to: T. Le Bertre, e-mail: Thibaut.LeBertre@obspm.fr
$2500 \mathrm{~K}$, hydrogen should be mainly molecular. It should stay molecular out to the point where it would be photodissociated $(E=4.5 \mathrm{eV})$ by the Interstellar Radiation Field (ISRF). On the other hand, for $T_{\star}>2500 \mathrm{~K}$, hydrogen should be mainly atomic. In both cases, hydrogen should be photo-ionized $(E=13.6 \mathrm{eV})$ in the most external parts of the circumstellar shells. But the real situation might be more complex, due to, e.g., the propagation of shocks, which could dissociate $\mathrm{H}_{2}$, or the formation of $\mathrm{H}_{2}$ on grains.

The atomic hydrogen $\mathrm{HI}$ line at $21 \mathrm{~cm}$ has been searched for in several evolved sources. Zuckerman et al. (1980) have carried out a search with the $305 \mathrm{~m}$ Arecibo antenna in the direction of 13 red giants, among which IRC +10216 , and planetary nebulae; none was detected. With the Very Large Array (VLA), Bowers \& Knapp (1987) could detect an emission from $\alpha$ Ori, but failed to detect IRC +10216 . Also with the VLA, Bowers \& Knapp (1988) detected $o$ Ceti (Mira) in emission. Finally, using the Westerbork Synthesis Radio Telescope (WSRT) Taylor et al. (1990) detected HI from 5 planetary nebulae, in absorption against a continuum emitted by the central ionized core. Their high rate of success (as compared to Zuckerman et al.) can be due to the selection of compact PNs and to the small size of the synthesized WSRT beam $\left(\sim 15^{\prime \prime}\right)$ as compared to the Arecibo beam $\left(3.2^{\prime}\right)$. Taylor et al. infer that the observed HI absorption comes from material lost by the central stars in previous phases of their evolution. This suggests that the winds from AGB stars should be at least partly atomic.

Although atomic hydrogen might be common in circumstellar shells, the HI line at $21 \mathrm{~cm}$ is difficult to detect because it is intrinsically weak, and also because $\mathrm{HI}$ is found everywhere in the Galaxy and produces an intense background which needs to be subtracted. So far, only one 
genuine AGB source has been detected, o Ceti; it has a spectral type M7IIIe which translates to an effective temperature $\sim 3000 \mathrm{~K}$ (Perrin et al. 1998). This could lend support to the Glassgold \& Huggins (1983) model. We note, however, that $o$ Ceti is known to have a hot companion, a white dwarf embedded in an accretion disk (Karovska 1999), which could contribute to $\mathrm{H}_{2}$ photo-dissociation. Similarly, $\alpha$ Ori, a supergiant of type M1-2 I, is known to have a strong UV-excess, possibly due to a chromosphere, which makes its wind mostly atomic.

\section{Observations}

IRC +10216 is the prototype of mass-losing carbon-rich AGB sources. Its distance, estimated from the periodluminosity relationship for carbon Miras (Groenewegen \& Whitelock 1996), is $\sim 135 \mathrm{pc}$ (Le Bertre 1997). Its present mass loss rate is of the order of $2 \times 10^{-5} M_{\odot} \mathrm{yr}^{-1}$ (TruongBach et al. 1991; Le Bertre 1997). It is surrounded by a shell expanding at a velocity of $\sim 15 \mathrm{~km} \mathrm{~s}^{-1}$. More than 50 molecular species have been detected and sometimes mapped (Lucas \& Guélin 1999). The emission is seen to extend out to $30^{\prime \prime}$ from the central star, which seems to indicate that beyond this limit the molecules are photo-dissociated by the ISRF. However, Groenewegen \& Ludwig (1998) could detect CO emission out to $110^{\prime \prime}$, indicating that CO is self-shielded (Mamon et al. 1988). The circumstellar envelope is certainly still much larger. Using IRAS data, Young et al. (1993) could trace the emission at $100 \mu \mathrm{m}$ up to $9.5^{\prime}$ from the central star. Furthermore, there are growing evidences that the matter around this source is not distributed smoothly. For instance Mauron \& Huggins (2000) found that the dust has an irregular radial distribution.

The central star is presently of late carbon type, C9,5 (Cohen 1979), with an effective temperature $\sim 2200 \mathrm{~K}$. It has no known companion. Therefore one would not expect hydrogen in atomic form close to the central star. On the other hand, the mass loss rate is considerable and the object is close to the Sun. Also, it is seen at a high galactic latitude $\left(b^{\mathrm{II}} \sim 45^{\circ}\right)$ and a low contamination from galactic H I emission can be expected. Finally, atomic carbon, C I at $610 \mu \mathrm{m}$, has been detected by Keene et al. (1993) with the Caltech Submillimeter Observatory (CSO); the emission seems to be extended $\left(\sim 30-90^{\prime \prime}\right)$. For these different reasons, we selected IRC +10216 as our first target.

The observations have been carried out during the first semester of the year 2001 with the upgraded Nançay Radiotelescope (NRT) (van Driel et al. 1996). The NRT is a transit instrument which can track a source for about one hour every day. At $21 \mathrm{~cm}$, the half-power beam-width is $4^{\prime}$ in the E-W direction and $22^{\prime}$ in the N-S direction. The beam efficiency (measured on the Moon) is 0.65 . The point source efficiency is $1.4 \mathrm{~K} \mathrm{Jy}^{-1}$ and the total sytem noise $\sim 35 \mathrm{~K}$.

We have tried both the frequency-switch and the position-switch modes. The latter proved to be more stable because the baseline fluctuations cancel better.

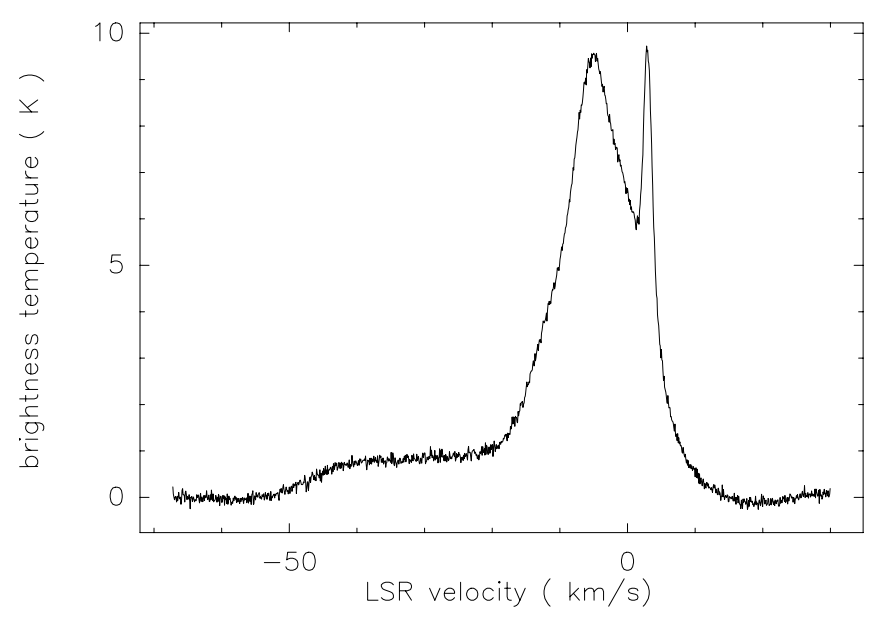

Fig. 1. On-source spectrum. This spectrum results from the sum of 18 spectra of $1 \mathrm{~min}$ acquired on 25 March 2001. The resolution is $0.04 \mathrm{~km} \mathrm{~s}^{-1}$ and the rms noise, $\sim 0.08 \mathrm{~K}$.

The 8192 channel autocorrelator was split in 4 banks to provide the vertical $\left(\mathrm{PA}=0^{\circ}\right)$ and horizontal $(\mathrm{PA}=$ $90^{\circ}$ ) linear polarisation, as well as the left-handed and right-handed circular polarisation vectors. Since the HI line is thermally excited, we only used the total intensity or Stokes parameter I. The spectral bandwidth was $80 \mathrm{~km} \mathrm{~s}^{-1}$ and centered on the stellar velocity of IRC $+10216, V_{\mathrm{LSR}}=-26 \mathrm{~km} \mathrm{~s}^{-1}$; the spectral resolution was $0.04 \mathrm{~km} \mathrm{~s}^{-1}$ (2048 channels).

An on-source spectrum obtained in the frequencyswitch mode is displayed in Fig. 1. The antenna temperatures have been converted to brightness temperatures. It shows mainly the local HI emission with an LSR-velocity close to $0 \mathrm{~km} \mathrm{~s}^{-1}$. The signal from IRC +10216 is expected to be centered on $V_{\mathrm{LSR}}=-26 \mathrm{~km} \mathrm{~s}^{-1}$ and to have a width of at most $2 \times 15 \mathrm{~km} \mathrm{~s}^{-1}$, i.e. twice the expansion velocity measured from the $\mathrm{CO}$ lines. In this spectral range, after subtraction of the baseline, the observed brightness temperature is $\sim 1.2 \mathrm{~K}$. Assuming a temperature $T_{\mathrm{i}}=50 \mathrm{~K}$ for the interstellar cloud responsible for this emission, its optical depth, $\tau_{\mathrm{i}}$, should be around 0.02 . We note however that above $-15 \mathrm{~km} \mathrm{~s}^{-1}$ the galactic $\mathrm{HI}$ emission spectrum rises steeply. Our observations agree with the results presented by Zuckerman et al. (1980).

In the position-switch mode, the data were acquired by doing cycles of $1 \mathrm{~min}$ on the source, then $1 \mathrm{~min}$ on the off-position at positive right ascension (RA), and finally 1 min on the off-position at negative RA. The off-positions were selected at \pm 1 NRT beam (i.e. $\left.\pm 4^{\prime}\right), \pm 2$ beams $\left( \pm 8^{\prime}\right)$, and \pm 3 beams $\left( \pm 12^{\prime}\right)$.

In Figs. 2-4, we present the resulting (on-off)spectra. The scatter in the spectra increases at $V_{\mathrm{LSR}}>$ $-10 \mathrm{~km} \mathrm{~s}^{-1}$ due to the rapidly varying galactic HI emission (see Fig. 1). Absorption features centered near $V_{\mathrm{LSR}}=-26 \mathrm{kms}^{-1}$ are clearly seen in the 3 cases. We stress that these spectra are entirely independent, the onspectra being acquired anew every time.

Reference spectra taken in the frequency-switch mode at $\pm 4^{\prime}$ and $\pm 8^{\prime}$ in RA and at $\pm 22^{\prime}$ in the N-S direction 


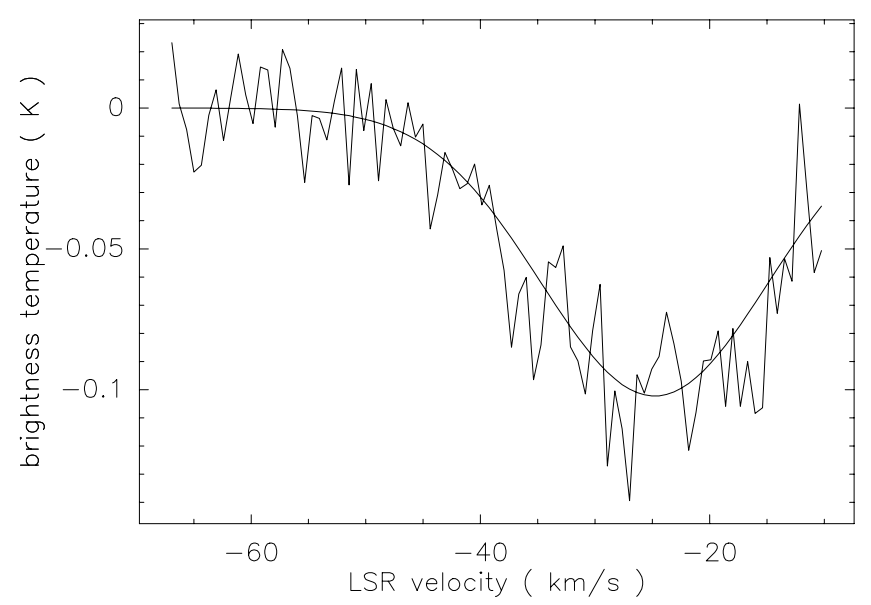

Fig. 2. (on-off) spectrum obtained on 29-30/03 and $1 / 04 / 2001$. The off-positions are placed at $\pm 4^{\prime}$ in right ascension from the on-source position, i.e. \pm 1 beam. The spectrum results from the sum of 32 on-spectra of 1 min and $2 \times 32$ offspectra of $1 \mathrm{~min}$. It is averaged with a boxcar of 16 channels.

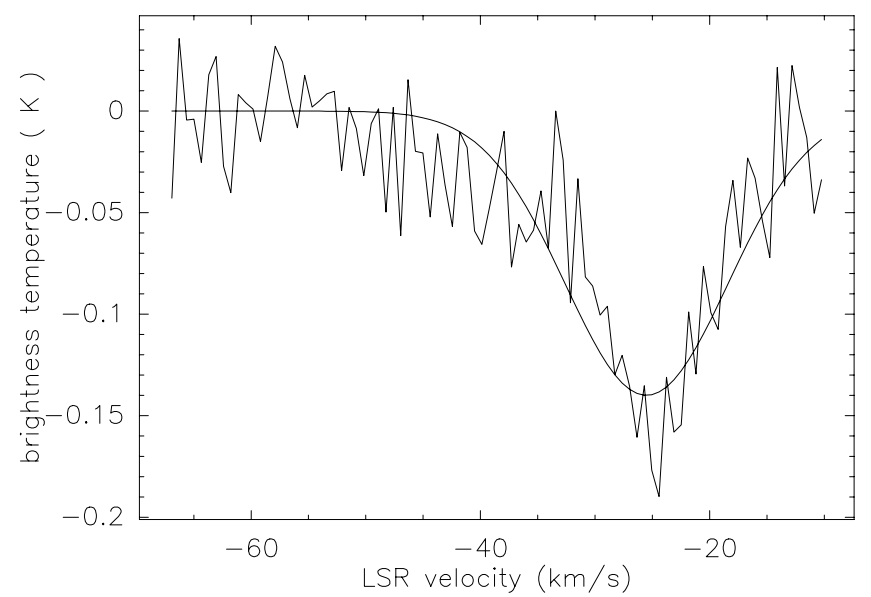

Fig. 3. Same as Fig. 2, but the off-positions are at $\pm 8^{\prime}$ (19 cycles acquired on $2-3 / 04 / 2001$ ).

(i.e. \pm 1 NRT beam) were averaged to improve the signal/noise ratio. The difference spectra show the same absorption feature near $-26 \mathrm{~km} \mathrm{~s}^{-1}$.

\section{Interpretation and discussion}

To characterize this absorption feature, we have fitted the difference spectra with Gaussian curves, excluding the velocity range $\left[-10,+5 \mathrm{~km} \mathrm{~s}^{-1}\right]$. The results are given in Table 1, first for the position-switch mode $(\mathrm{P} / \mathrm{S})$, then for the frequency-switch mode $(\mathrm{F} / \mathrm{S})$. The absorption depends on the selection of the reference positions. It is deeper and narrower with the off-positions at $\pm 8^{\prime}$ and $\pm 12^{\prime}$ (and $\pm 22^{\prime}$ ) than with those at $\pm 4^{\prime}$. This effect indicates that the $\mathrm{HI}$ absorption comes from an extended region of diameter $\sim 12^{\prime}$, much larger than the region of C I emission $\left(\sim 1.5^{\prime}\right.$, Keene et al. 1993). In the following we estimate that the source is diluted in the NRT beam $\left(4^{\prime} \times 22^{\prime}\right)$ by a factor 1.8. Since Zuckerman et al. (1980) made offsource measurements on four positions displaced by only $\pm 3.5^{\prime}$ in RA and Dec, they could hardly detect such an

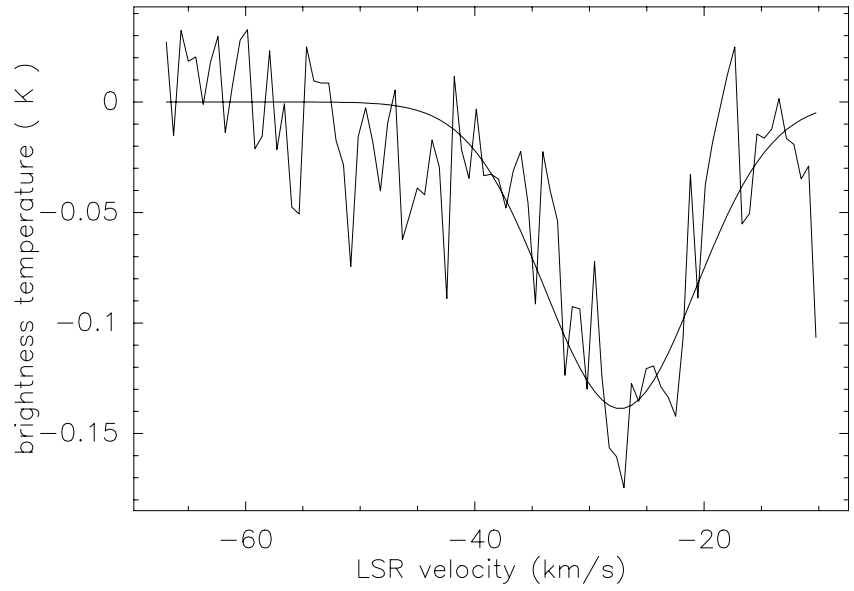

Fig. 4. Same as Fig. 2, but the off-positions are at $\pm 12^{\prime}$ (25 cycles acquired on $4-5-6 / 04 / 2001)$.

Table 1. Results of the Gaussian fits to the H I line profiles. The rms noise $(\sigma)$ is given for a spectral element of $0.64 \mathrm{~km} \mathrm{~s}^{-1}$.

\begin{tabular}{lccccc}
\hline off-positions & $\begin{array}{c}V_{\text {cent. }} \\
\mathrm{km} \mathrm{s}^{-1}\end{array}$ & $\begin{array}{c}F W H M \\
\mathrm{~km} \mathrm{~s}^{-1}\end{array}$ & $\begin{array}{c}F_{\text {peak }} \\
\mathrm{Jy}\end{array}$ & $\begin{array}{c}\sigma \\
\mathrm{Jy}\end{array}$ & Fig. \\
\hline $\mathrm{P} / \mathrm{S}$ & & & & & \\
$\pm 4^{\prime} \quad(\mathrm{E}-\mathrm{W})$ & -24.7 & 23.3 & -0.10 & 0.025 & 2 \\
$\pm 8^{\prime} \quad(\mathrm{E}-\mathrm{W})$ & -25.5 & 16.7 & -0.14 & 0.030 & 3 \\
$\pm 12^{\prime} \quad(\mathrm{E}-\mathrm{W})$ & -27.2 & 15.5 & -0.14 & 0.035 & 4 \\
\hline $\mathrm{F} / \mathrm{S}$ (see text) & -27.0 & 12.5 & -0.12 & 0.025 & \\
\hline
\end{tabular}

absorption. Also, using the VLA with a minimum spacing of $80 \mathrm{~m}$, Bowers \& Knapp (1987) could not be sensitive to a structure larger than $9^{\prime}$.

We now examine the condition for observing $\mathrm{HI}$ in absorption. For simplicity we assume that H I is always optically thin; we already checked that this is correct for the interstellar emission $\left(\tau_{\mathrm{i}} \approx 0.02\right)$. We also assume that the H I interstellar cloud is located beyond IRC +10216 . The hydrogen in the source is at a temperature, $T_{\mathrm{s}}$, and has an optical depth, $\tau_{\mathrm{s}}$. In these simplified conditions:

$$
\begin{aligned}
T_{\mathrm{on}} & \approx\left(T_{\mathrm{CB}}+\tau_{\mathrm{i}} T_{\mathrm{i}}\right) \times\left(1-\tau_{\mathrm{s}}\right)+\tau_{\mathrm{s}} T_{\mathrm{s}} \\
T_{\text {off }} & \approx T_{\mathrm{CB}}+\tau_{\mathrm{i}} T_{\mathrm{i}} \quad \text { (out of the source) } \\
\Delta T & \approx-\tau_{\mathrm{s}} \times\left(T_{\mathrm{CB}}+\tau_{\mathrm{i}} T_{\mathrm{i}}\right)+\tau_{\mathrm{s}} T_{\mathrm{s}} \\
& \approx \tau_{\mathrm{s}} \times\left(T_{\mathrm{s}}-T_{\mathrm{CB}}-\tau_{\mathrm{i}} T_{\mathrm{i}}\right) .
\end{aligned}
$$

Therefore, to observe a feature in absorption, we should have:

$T_{\mathrm{s}}<T_{\mathrm{CB}}+\tau_{\mathrm{i}} T_{\mathrm{i}} \approx 2.7 \mathrm{~K}+1.2 \mathrm{~K}=3.9 \mathrm{~K}$.

This upper limit is conservative, as it is likely that part of the $\mathrm{HI}$ interstellar emission arises in the foreground of IRC +10216 .

It is difficult to extract a column density corresponding to the observed absorption feature because the temperature and the optical depth are strongly correlated (Levinson \& Brown 1980). If we assume $T_{\mathrm{s}} \approx 3 \mathrm{~K}$, then, from the observed H I absorption, $\tau_{\mathrm{s}} \approx 0.2$, and we can derive a column density:

$N_{\mathrm{HI}} \sim 1.8 \times 10^{18} \tau_{\mathrm{s}} T_{\mathrm{s}} \Delta V \approx 2 \times 10^{19} \mathrm{~cm}^{-2}$. 
If we assume that the neutral atomic hydrogen is in a shell of internal radius $2^{\prime}$ and external radius $6^{\prime}$, and that the mass loss has been continuous with a constant velocity of $15 \mathrm{~km} \mathrm{~s}^{-1}$ :

$\dot{N}_{\mathrm{HI}} \sim 3.4 \times 10^{24} N_{\mathrm{HI}} \approx 7 \times 10^{43} \mathrm{~s}^{-1}$

or:

$\dot{M}_{\mathrm{HI}} \sim 0.9 \times 10^{-25} N_{\mathrm{HI}} \approx 2 \times 10^{-6} M_{\odot} \mathrm{yr}^{-1}$.

This estimate is reasonable in view of the present mass loss rate $\left(2 \times 10^{-5} M_{\odot} \mathrm{yr}^{-1}\right)$. But it should not be overemphasized as it relies on a uniform temperature for the HI layer, with an arbitrary value. If we had adopted $T_{\mathrm{s}} \approx 3.5 \mathrm{~K}$ (resp. $\left.2.0 \mathrm{~K}\right)$, then we would have got $\dot{M}_{\mathrm{HI}} \approx$ $5 \times 10^{-6} M_{\odot} \mathrm{yr}^{-1}$ (resp. $5 \times 10^{-7} M_{\odot} \mathrm{yr}^{-1}$ ). The dilution factor that we have adopted (1.8) is also uncertain, but numerical simulations indicate that it should not affect our estimates by more than a factor of 2 . A better mapping would be useful to characterize the HI shell responsible for the observed absorption.

The gas in the expanding circumstellar shells is certainly cooling down to very low temperatures, e.g. 5-10 K at $\sim 0.15 \mathrm{pc}$ (Kwan \& Hill 1977). However, the upper limit to the temperature that we derive seems extremely low. We note that Truong-Bach et al. (1991) needed to use kinetic temperatures lower that those of Kwan \& Hill (1977) to fit their $\mathrm{CO}$ data. Also, a recent and complete modelling of the IRC +10216 CO shell has been performed by Groenewegen et al. (1998); they derive a temperature profile which shows a minimum, in the range 4 to $6 \mathrm{~K}$ for their preferred models, at $\sim 3 \times 10^{17} \mathrm{~cm}$, but still above our upper limit. On the other hand, Sahai (1990) claims that, in sources with large mass loss rate $\left(>10^{-5} M_{\odot} \mathrm{yr}^{-1}\right)$, there could exist an external layer with very low kinetic temperature, below $2.7 \mathrm{~K}$, as a result of the wind adiabatic expansion and of a reduced grain photo-electric heating.

Patzer et al. (2000) have developed a non-equilibrium photo-chemical model of the IRC +10216 circumstellar shell. The initial conditions are fixed at the photosphere. From there, the gas composition of the expanding wind is followed as a function of the distance to the central star. Their model is in good agreement with the observations although the sizes of some emissions might be underestimated due to the neglect of self-shielding; for instance they find that $\mathrm{C}$ I should be found between $10^{16} \mathrm{~cm}\left(5^{\prime \prime}\right)$ and $5 \times 10^{16} \mathrm{~cm}\left(25^{\prime \prime}\right)$. In this model most of the hydrogen should be in H I from $2 \times 10^{16} \mathrm{~cm}\left(10^{\prime \prime}\right)$ to $10^{18} \mathrm{~cm}\left(\sim 8^{\prime}\right)$. These 2 limits correspond to the photo-dissociation of $\mathrm{H}_{2}$ and to the photo-ionization of H I by the ISRF. Therefore the H I line that we observed could be due to the photodissociation of $\mathrm{H}_{2}$ in the exterior of the circumstellar shell. On the other hand, Patzer et al. find a kinetic temperature $\sim 15 \mathrm{~K}$ at $10^{18} \mathrm{~cm}$, which is too large to explain that the line is seen in absorption.

At a distance of $135 \mathrm{pc}$, the beam of the NRT is sampling a zone of $0.16 \mathrm{pc} \times 0.86 \mathrm{pc}$. The material which is now located at $0.1 \mathrm{pc}$ from the central star was expelled by it $\geq 6 \times 10^{3}$ years ago. This lapse of time is not small as compared to the timescale for stellar evolution on the TPAGB, the separation between 2 thermal pulses being on the order of $10^{4}-10^{5}$ years. During this period the effective temperature of the central star could have been larger than presently and its outflowing wind, mostly atomic. The H I absorption that we have detected could then be the trace of an old $\left(\geq 6 \times 10^{3}\right.$ years $)$ atomic wind.

\section{Conclusion}

H I in IRC + 10216 has been detected in absorption against the cosmic background and possibly against interstellar H I emission coming from a galactic cloud located further away. This result indicates that the temperature in the external parts of circumstellar shells may reach lower values than commonly expected, giving support to the claim of Sahai (1990). It supports also the conclusion of Taylor et al. (1990) that H I should be common in stellar winds from AGB stars. The atomic hydrogen could result from the photo-dissociation of molecular hydrogen or be a remnant of an old $\left(\geq 6 \times 10^{3}\right.$ years $)$ atomic wind.

Acknowledgements. We are grateful to Dr. Beate Patzer for useful discussions.

\section{References}

Bowers, P. F., \& Knapp, G. R. 1987, ApJ, 315, 305

Bowers, P. F., \& Knapp, G. R. 1988, ApJ, 332, 299

Cohen, M. 1979, MNRAS, 186, 837

Glassgold, A. E., \& Huggins, P. J. 1983, MNRAS, 203, 517

Groenewegen, M. A. T., \& Ludwig, H.-G. 1998, A\&A, 339, 489

Groenewegen, M. A. T., van der Veen, W. E. C. J., \& Matthews, H. E. 1998, A\&A, 338, 491

Groenewegen, M. A. T., \& Whitelock, P. A. 1996, MNRAS, 281,1347

Karovska 1999, IAU Symp., 191, 139

Keene, J., Young, K., Phillips, T. G., Büttgenbach, T. H., \& Carlstrom, J. E. 1993, ApJ, 415, L131

Kwan, J., \& Hill, F. 1977, ApJ, 215, 781

Le Bertre, T. 1997, A\&A, 324, 1059

Levinson, F. H., \& Brown, R. L. 1980, ApJ, 242, 416

Lucas, R., \& Guélin, M. 1999, IAU Symp., 191, 305

Mamon, G. A., Glassgold, A. E., \& Huggins, P. J. 1988, ApJ, 328,797

Mauron, N., \& Huggins, P. J. 2000, A\&A, 359, 707

Olofsson, H. 1999, IAU Symp., 191, 3

Patzer, A. B. C., Winters, J. M., \& Sedlmayr, E. 2000, IAU Symp., 177, 573

Perrin, G., Coudé du Foresto, V., Ridgway, S. T., et al. 1998, A\&A, 331, 619

Sahai, R. 1990, ApJ, 362, 652

Taylor, A. R., Gussie, G. T., \& Pottasch, S. R. 1990, ApJ, 351, 515

Truong-Bach, Morris, D., Nguyen-Q-Rieu 1991, A\&A, 249, 435 van Driel, W., Pezzani, J., \& Gérard, E. 1996, in High Sensitivity Radio Astronomy, ed. N. Jackson, \& R. J. Davis (Cambridge Univ. Press), 229

Young, K., Phillips, T. G., \& Knapp, G. R. 1993, ApJS, 86, 517

Zuckerman, B., Terzian, Y., \& Silverglate, P. 1980, ApJ, 241, 1014 